\title{
Review on Routing Techniques in Wireless Sensor Networks
}

\author{
Amanpreet Singh \\ Post Graduate Student \\ Chandigarh Engg College \\ Landran, India
}

\author{
Rohit Bajaj, Ph.D. \\ Associate Professor \\ Chandigarh Engg College \\ Landran, India
}

\begin{abstract}
The popularity of wireless sensor networks (WSN) in the various applications related to weather monitoring, activity monitoring, fire monitoring, underwater application, snow \& avalanche studies, etc. The WSN sensors are battery running devices, hence prone to the energy efficiency problems. There are several energy efficient solutions in existence which optimizes routing cost/metric calculation, load balancing approaches, data forwarding applications, etc. This paper proposes a survey on such energy efficient approaches for WSNs. The tree based routing, single path or multi path routing, optimized metric routing, etc have been evaluated under this performance evaluation survey. The tree based approaches are considered better approaches than other in terms of effectiveness. The tree based approaches offer the use maximum lifetime in the WSNs.
\end{abstract}

\section{General Terms}

Routing Algorithm, Path forwarding, Energy consumption, Energy-aware routing.

\section{Keywords}

Survey, routing, WSN survey, route metric, energy efficiency.

\section{INTRODUCTION}

The demand for wireless technology has increased rapidly because of its low cost implementation and easy maintenance. Initially the applications of wireless were limited to research and industrial purposes which are now attracting areas of personal use as well. The technology that is emerging rapidly out of the wireless infrastructure is the Wireless Sensor Networks (WSN's). The major driving force behind the increasing demand of WSN is because of its ability to operate in harsh environments with an increased level of performance in comparison to any other technology.

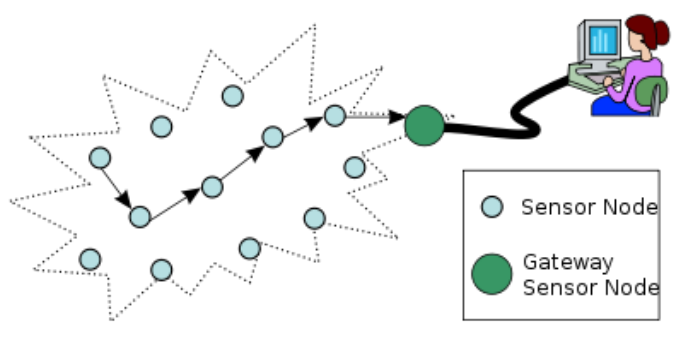

Figure 1: WSN Architecture

WSN consists of very small sensor nodes deployed in the area to be observed. These sensor nodes are smart sensors that record any activity within their area of reach without human interaction and report back to the sink node or base station that collectively process the data obtained from multiple sensor nodes. Being small, sensor nodes can be easily deployed and are good at seeing things that we humans directly can't. The sink node inside the wireless sensor network acts as a gateway that connects the sensor nodes to the outside wired internet world, allowing the users at far by locations to easily monitor the activities by the use of sensors. Such ease of access makes WSN applicable in broad areas alongside defining limited boundaries such as moving objects (vehicles) or opening/closing of doors within a building that hinder the signal strength of a wireless network. To overcome such issues we need an intelligent routing technique.

\section{ROUTING}

Routing is a Network Layer protocol that decides the transmission route for any packet. Since, each and every sensor node in the wireless sensor network is powered by disposable batteries, routing becomes a challenging task where a best route is to be selected that is free from dynamic objects and ultimately results in increased network lifetime. In WSN, selecting a best path is not enough, the routing protocol needs to be intelligent enough to tackle node failures and quickly overcome those failures by identifying new routes to transfer data.

The two major categories of routing protocols are :

1. Distance-Vector Routing Protocol

2. Link-State Routing Protocol

\subsection{Distance Vector Routing Protocol}

It makes use of Belman-Ford and Ford-Fulkerson algorithm to calculate transmission paths. In this algorithm, each node maintains a vector table of minimum distance to every node and shares their table with neighboring nodes which in-turn compare it with their own table to find the best route. The major drawback of this protocol is that it consumes a lot of bandwidth as they pass the entire routing table and is therefore slower. Moreover, if there is any topology change then only the immediate neighbors of the current node are informed while in other type of routing protocol (link-state), all the nodes in the network are informed about the topology change.

\subsection{Link State Routing Protocol}

It is another type of routing protocol. Unlike distance-vector, link state never pass the entire routing table instead the current node shares only information about its directly connected links to the entire network. From this point, it can be said that in link-state routing every node is aware of the entire network topology, which in-result makes the network harder to configure.

\section{TREE BASED ROUTING}

Tree based routing is a new type of protocol that covers all the issues that are left by both distance vector and link based routing protocols. The major purpose of tree-based routing is to tackle route instability by introducing neighborhood 
heuristics technique which involves a combination of present sensor node's routing metrics and the metrics of its neighbors to identify quality routes and other alternatives at the time of primary route failure. As a result, the sensors with multiple routing choices are selected for forwarding the packets. This protocol is well suited for low power networks.

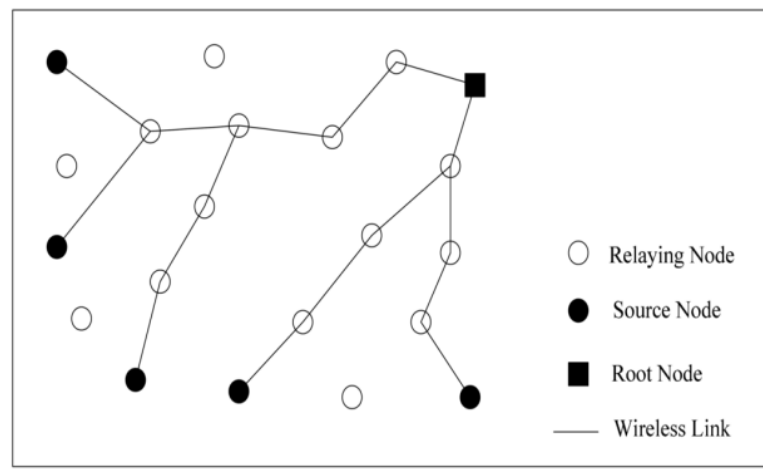

Figure 2: Tree Routing Structure

In Tree-Based network sink node is the central top node where all the data is collected and is referred to as root node. The sensors as leaf node sense the data from their surroundings as pass it on to the nodes above them as parent nodes which further forwards the data till sink or root node. Moreover, the sensor nodes as leaf nodes choose the nodes with good quality alternative routes as their parent nodes so that when the parent's primary route fails it can easily route the data through another neighbor route.

\subsection{Issues in Tree-based Routing}

In Tree-Based routing, neighborhood metric calculation mechanism was used to highlight the best path to forward the data in order to reduce network instability and increase the network lifetime but the energy level evaluation for metric calculation is not done in the present tree-based routing algorithm which may result in instability at some extent. There is a strong need of load-balancing so that data can be transferred in the parallel manner at high bandwidth through shorter routes with balanced energy.

The tree-based routing require connection pruning to prevent the routing loops. The tree based connectivity can cause multiple links to the similar path, or non similar path, which can cause the data moving the infinite routing loop where data keep moving in the network until the Time to live (TTL) is over. This leaves the whole network in ruckus and jams the operations of the WSN cluster. This can be overcome by pruning the links which can cause the routing loops in the WSN cluster.

The tree based routing protocols are prone to the energy efficiency based problems. The tree has a parent connected with its branches. The branch nodes if higher in density, can put heavier load on the parent node, which cause higher energy consumption and leads to its shutdown due to low energy. This leaves the entire cluster out of the connectivity, which may produce the hazardous situations by producing the information gaps in the sensitive information areas.

\section{RELATED WORK}

Delaney,et.al. [1] developed a routing framework that is reliable for tree-based routing architecture in WSN that involves the idea of Neighbor Heuristics $(\mathrm{NH})$, a mechanism that integrates sensor routing metric with its adjoining nodes which focus on the present route aspects and the kind of routing choices feasible to sensor so that best alternate can be applied when its current route becomes distant.

Ghadimi, et.al. [2] proposed an efficient opportunistic routing scheme for WSN i.e ORW which needs a different opportunistic routing metric ,EDC that indicate the expected number of duty-cycled wakeups which are necessary to transfer a packet from origin to destination.

Sahin, et.al. [3] examined the purpose of single path and multi path QoS aware routing algorithm under rigid situation for assessment of their service differentiation capacity in reliable and timeliness fields.

Singh, et.al. [4] developed an energy efficient tree-based routing technique with timestamp in which parent-child relation is used to send the packet. The source that broadcasts the packet should arrive at target in pre-specified time.

Kwon, et.al. [5]proposed a stateless point to point routing protocol that depends on the shortcut tree routing algo which transfer packet to node with minimal remaining hop-count between the neighboring nodes without any extra control overhead.

Tunca, et.al. [6]presented a survey of current distributed mobile sink routing protocols that discusses the arrangement and objection combined with difficulties of mobile sink routing that are determined and made clear.

Bechkit, et.al. [7]proposed a unique weighted shortest path tree that converge cast traffic routing in WSN which presents anew weighted path cost function and exhibits how this cost function is more relevant for WSN. An adequate Weighted shortest path tree is created with this cost function and does not insert a new overhead.

BartoszMusznicki et.al.[8] presented Dijkstra based localized multicast routing in WSN (DLEMA) which targets on exploring the energy shortest path popular through nodes that support best geographical advance toward destination.

Riham S Elhabyan, et.al.[9] proposed two different routing protocols that are Control Data Disemmination Protocol(CDDP) which arranges tree that links all the nodes in the network which will transmit control data to base station. The second is centralized weighted clustering protocol that uses CDDP for transmission of control data to base station and will prefer the leading cluster heads in network according to weighted criteria function.

Huaiyu Wen, .et.al.[10] presented a load balance routing protocol that is based upon cross-layer knowledge for wireless mesh network and adopts adhoc on demand distance vector routing protocol as prototype named as Load Balance Cross layer AODV (LBCL-AODV).the LBCL-AODV considers hop counts and cross layer information is used as metric to enhance route options with the help of node load and packet delivery rate performance parameters.

Marc Barcelo, .et.al[11] presented an alternate routing metric for CDP, mentioned as MaxPDR that comprises a transmission power control in routing process. MaxPDR has been enforced in marketing motes to assess its performance and contrasted it with routing mertices that are used by first CTP and Zigbee.

HamzehAljawawdeh, .et.al[12] proposed Dynamic load balancing protocol for WSN that accomplish all the nodes in network to obtain load balance and extend WSN lifetime. DLBP flourished to form a load balance tree that excludes the demand of control messages during routing of data,send 
messages to next hop. Support and fix network errors and loss instantly.

\section{CONCLUSION AND FUTURE WORK}

This theoretical critical analysis was conducted on various routing WSN techniques. The primary focus of this survey lied on the problem of energy efficiency in the WSN routing. The various routing techniques have been evaluated under this literature survey to find the best technique or type of techniques for the energy efficiency in the WSN routing process. This survey has followed the all new approach for the critical analysis of the existing routing schemes on the basis of their effectiveness in path forwarding accuracy and energy consumption. The survey has resulted that the tree based routing approaches are the most efficient techniques among all other routing techniques in terms of energy efficiency. The energy efficient tree based routing is successful because of its segmented connectivity design. The tree-based routing scheme connects the nodes in the tree topology, which is termed as the layered approach for WSN routing. The tree based topology divides the network load in the small or medium sized networks, but not effective with the larger sized networks because there can be many nodes in the tree topology with high network load.The tree based routing mechanisms uses the connectivity tree for the interconnectivity of the nodes, and to deliver the data towards the sink. The tree based routing can be optimized as single path or multi path routing mechanism. In the future, the existing tree based routing techniques can be improved for higher their efficiency than the existing ones. Also a more detailed survey can be performed on the tree based routing by implementing some of the techniques by simulation them in the real-time on a uniform WSN scenario.

\section{REFERENCES}

[1] Delaney, D., Russell Higgs, and G. O'Hare. "A stable routing framework for tree-based routing structures in wsns." (2014): 1-1.Gayan, Samiru, and Dileeka Dias. "Improved DV-Hop algorithm through anchor position re-estimation." In Wirelessand Mobile, 2014 IEEE Asia Pacific Conference on, pp. 126-131. IEEE, 2014.Tavel, P. 2007 Modeling and Simulation Design. AK Peters Ltd.

[2] Ghadimi, Euhanna, Olaf Landsiedel, Pablo Soldati, Simon Duquennoy, and Mikael Johansson. "Opportunistic Routing in Low Duty-Cycled Wireless Sensor Networks." ACM Transactions on Sensor Networks 10, no. 4 (2014).

[3] Sahin, Dilan, VehbiCagriGungor, TaskinKocak, and Gurkan Tuna. "Quality-of-service differentiation in single-path and multi-path routing for wireless sensor network-based smart grid applications." Ad Hoc Networks (2014).Li, Mo, and Yunhao Liu. "Rendered path: range-free localization in anisotropic sensor networks with holes." In Proceedings of the 13th annual ACM international conference on Mobile computing and networking, pp. 51-62. ACM, 2007.

[4] Singh, Dharmendra, Shubhanjali Sharma, Vinesh Jain, and JyotiGajrani. "Energy efficient source based tree routing with time stamp in WSN." In Signal Propagation and Computer Technology (ICSPCT), 2014 International Conference on, pp. 120-124. IEEE, 2014.Liu, Yong, Yu $\mathrm{Hen} \mathrm{Hu}$, and Quan Pan. "Distributed, robust acoustic source localization in a wireless sensor network." Signal Processing, IEEE Transactions on 60, no. 8 (2012): 4350-4359.

[5] Kwon, Kiwoong, Minkeun Ha, Taehong Kim, SeongHoon Kim, and Daeyoung Kim. "The stateless point to point routing protocol based on shortcut tree routing algorithm for IP-WSN." In Internet of Things (IOT), 2012 3rd International Conference on the, pp. 167-174. IEEE, 2012.Bal, Mert, Henry Xue, WeimingShen, and Hamada Ghenniwa. "A Test-Bed for Localization and Tracking in Wireless Sensor Networks." In SMC, pp. 3581-3586. 2009.

[6] Tunca, Can, SinanIsik, M. Donmez, and CemErsoy. "Distributed Mobile Sink Routing for Wireless Sensor Networks: A Survey." (2014): 1-21.

[7] Bechkit, Walid, MouloudKoudil, YacineChallal, AbdelmadjidBouabdallah, BrahimSouici, and KarimaBenatchba. "A new weighted shortest path tree for convergecast traffic routing in WSN." In Computers and Communications (ISCC), 2012 IEEE Symposium on, pp. 000187-000192. IEEE, 2012.Kumar, Anil, ArunKhosla, Jasbir Singh Saini, and Satvir Singh. "Meta-heuristic range based node localization algorithm for Wireless Sensor Networks." InLocalization and GNSS (ICL-GNSS), 2012 International Conference on, pp. 1-7. IEEE, 2012.

[8] BartoszMusznicki, MikołajTomczak and PiotrZwierzykowski. "Dijkstra-based Localized Multicast Routing in Wireless Sensor Networks". In Networks and Digital Signal Processing , IEEE-2012, 8th IEEE, IET International Symposium on Communication Systems.Salman, Naveed, MounirGhogho, and A. Kemp. "Optimized low complexity sensor node positioning in wireless sensor networks." (2014): 1-1.

[9] Kiwoong Kwon, Minkeun Ha, Taehong Kim, SeongHoon Kim, and Daeyoung Kim. "The Stateless Point to Point Routing Protocol based on Shortcut Tree Routing Algorithm for IP-WSN", IEEE 2012.

[10] Riham S. Elhabyan, and Mustapha C.E. Yagoub, Senior Member, IEEE. "WEIGHTED TREE BASED ROUTING AND CLUSTERING PROTOCOL FOR WSN", 2013 26th IEEE Canadian Conference Of Electrical And Computer Engineering (CCECE).

[11] Huaiyu Wen and GuangChunLuo. "Load Balance Routing Protocol in Wireless Mesh Network based on Cross-layer knowledge", 2013 International Conference on Computational and Information Sciences.

[12] Marc Barcel, Alejandro Correa, Jos L pez Vicario and AntoniMorell. "Joint Routing and Transmission Power Control for Collection Tree Protocol in WSN", 013 IEEE 24th International Symposium on Personal, Indoor and Mobile Radio Communications: Mobile and Wireless Networks.

[13] HamzehAljawawdeh and ImanAlmomani, Member IEEE. "Dynamic Load Balancing Protocol (DLBP) for Wireless Sensor Networks", 2013 IEEE Jordan Conference on Applied Electrical Engineering and Computing Technologies (AEECT). 